\title{
Licensed Shared Access in Distributed Antenna Systems Enabling Network Virtualization
}

\author{
Ying $\mathrm{He}^{*}$, Eryk Dutkiewicz*, Gengfa Fang*, Markus Dominik Mueck ${ }^{\dagger}$ \\ *Department of Engineering, Macquarie University, Australia. \\ ${ }^{\dagger}$ Intel Mobile Communications, Germany. \\ Email:\{y.he, eryk.dutkiewicz, gengfa.fang\}@mq.edu.au, markus.dominik.mueck@intel.com
}

\begin{abstract}
A distributed antenna system (DAS) architecture is considered to be a key enabler for further Network Virtualization where different network configurations are created as needed by a centralized decision making unit that is typically integrated into the Cloud Radio Access Network (C-RAN) which offers a potential architecture for 5G wireless communication systems. Many schemes have been proposed for Fractional Frequency Reuse (FFR) for resource allocation in the static cellular network architecture. In this paper, we investigate using the emerging Licensed Shared Access (LSA) on the downlink cell edge in a Network Virtualization context. We derive a threshold of the LSA bandwidth ratio for the average capacity and analyze the average capacity gain. This provides a guide in the decision making for using LSA bandwidth in DAS with Network Virtualization.
\end{abstract}

Keywords-Licensed Shared Access, Distributed Antenna Systems, Fractional Frequency Reuse, Network Virtualization.

\section{INTRODUCTION}

A Distributed Antenna System (DAS) is considered to be one of the mainstream technologies for the next generation wireless communication, especially for the cell edge users in cellular networks. The seminal work of DAS was proposed in [1] and applications were used in improve the coverage of the indoor cellular networks and to reduce the outage without building extra base stations. Subsequently the concept was introduced into the cellular network by deploying multiple antennas in different sectors to improve performance within one cell or one macro cell [2].

Recently, the Cloud Radio Access Network (C-RAN), proposed in [3] and [4], has become a technology of great interest due to its efficiency and flexibility for future cellular networks. All signal processing and computing is performed in a central unit, such as a super base station. Previous deployed base stations become remote antenna units connected to the central unit through fiber with an RF switch. Since they are geographically separated they can be employed as antennas in DAS in the cellular network.

The Network Virtualization concept [5] introduces a high degree of configuration flexibility by sharing and dividing the resources into slices and allocate to streams. Cloud computing and centralized processing solve the cooperation difficulties caused by the large amount of information that needs to be otherwise exchanged between base stations on the backhaul. These features show the potential in improving cell edge users performance [6]. With the flexibility provided by C-RAN and Network Virtulization, how to allocate the resources utilizing a limited spectrum to achieve higher capacity becomes a key problem and the motivation of this paper.

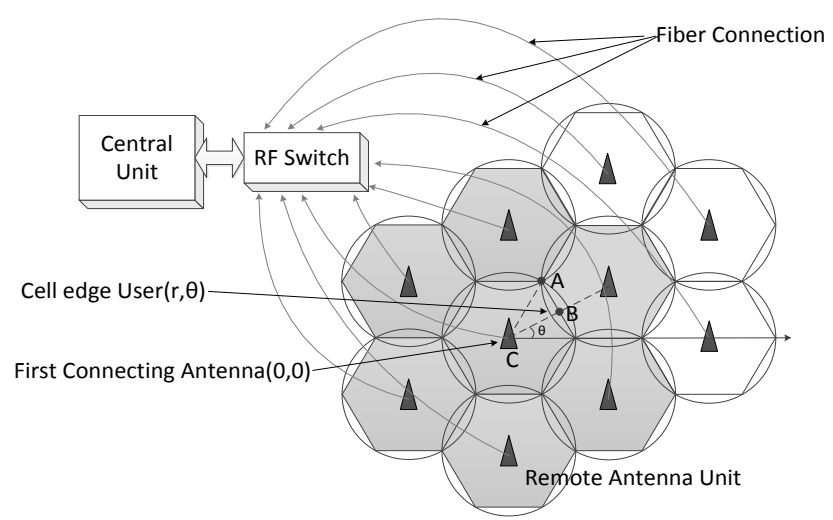

Fig. 1: Cloud Radio Access Network Architecture.

Due to the potential advantages of DAS and C-RAN, many mobile system operators are considering to use those technologies in current and next generation wireless communication networks, i.e. 4G [7] and 5G networks [8].

For 5G networks, a new Licensed Shared Access (LSA) concept has created much interests [9]. The LSA concept introduces spectrum sharing between an incumbent spectrum user and a Mobile Network Operator (MNO) user following a certain agreement. LSA shows potentials on increasing capacity of MNO users by obtaining more frequency resources [10]. Thus investigating how LSA will perform in DAS and C-RAN systems with Network Virtualization for 5G networks is the motivation of our paper. However, LSA is a novel approach and to the best of our knowledge the problem of FFR resource allocation in DAS for cell edge users in a dynamic Network Virtualization context has not been fully investigated yet. Although the LSA concept was presented in [11][12], no directly comparable work has been found in literature.

In our previous paper [6], we analyzed and compared the downlink capacity in C-RAN with DAS model using FFR. In this paper, we build the system model according to [6] and extend the results by adding usage of LSA in one cell.

The rest of the paper is organized as follows. Section II provides the system model of DAS and C-RAN. LSA capacity is analyzed and compared with MNO capacity in Section III. Numerical results are shown and discussed in Section IV. Finally, conclusions are drawn in Section V.

Notation: $E[\cdot]$ denotes the expectation operator. 


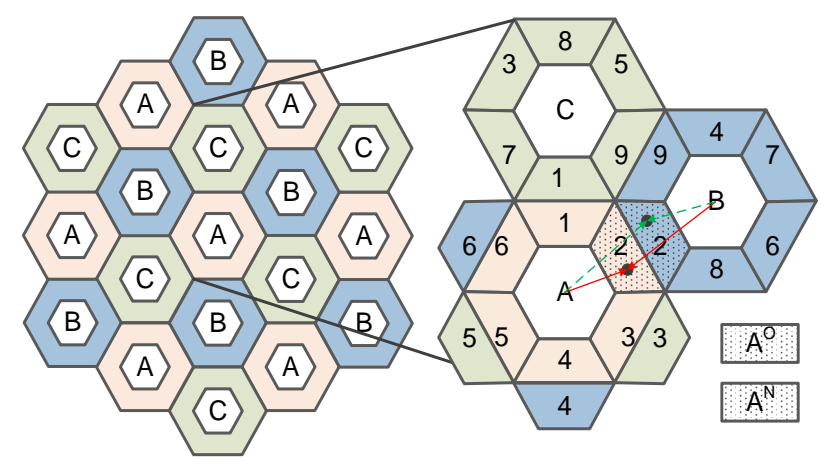

Fig. 2: MU-MIMO Fractional Frequency Reuse Pattern.

\section{SySTEM Model}

Our cellular network model setup is a C-RAN multicell and multiuser system including hexagonal shaped cells with one antenna in the center of each cell and mobile users in each cell. All the antennas are geographically separately located and connected through fiber to a cloud central unit, as illustrated in Fig. 1.

Assume that users are uniformly located in each cell, the user number is equal for each cell. In the Network Virtualization context, the concept of sharing is by dividing resource into slices. In this paper, the sharing of the downlink bandwidth is by dividing the bandwidth into frequency slices. Assume each slice has same bandwidth and for each user there is another user occupying the same bandwidth in the interfering cell. The bandwidth of each frequency slice is given as :

$$
\frac{B W_{\text {edge }}}{N_{f}}=\frac{B W_{\text {all }}-B W_{\text {center }}}{N_{f}}
$$

where $B W_{\text {edge }}$ is the bandwidth at the cell edge, $B W_{\text {center }}$ is the bandwidth in the cell center and $N_{f}$ is the frequency division number. We assume that the size of the cell center is $2 / 3$ of the cell radius. In order to increase the capacity for cell edge users, we use multi-user multiple-input and multipleoutput (MU-MIMO) transmission for multi-user cooperation and single-user multiple-input and single output (SU-MISO) for single user transmission with power gain [6].

\section{A. MU-MIMO using Coordinated Beamforming}

Consider MU-MIMO transmission, two antennas are transmitting to two users with different beams. We use CoMP-CB to accomplish MU-MIMO transmission, thus the receiving signal matrix for a pair of coodinated users is given by:

$$
\mathbf{y}=\sqrt{P_{t}} \mathbf{H} \mathbf{x}+\sum_{i \in B_{I}} \sqrt{P_{t}} \mathbf{H}_{i} \mathbf{x}_{i}+\mathbf{n}
$$

where $\mathbf{x} \in \mathbb{C}_{2 \times 1}^{T}$ is the transmitting signal from the remote antenna units, $\mathbf{H} \in \mathbb{C}_{2 \times 2}$ is the channel fading matrix, $\mathbf{x}_{i} \in \mathbb{C}_{2 \times 1}^{T}$ is the transmitting signal from the other remote antenna units to interfering users, $\mathbf{H}_{i} \in \mathbb{C}_{2 \times 2}$ is the channel fading matrix to interfering users, $\mathbf{n} \in \mathbb{C}_{2 \times 1}^{T}$ is the the Additive White Gaussian Noise (AWGN) of power density equals to $N_{0}$ and $\mathbf{y} \in \mathbb{C}_{2 \times 1}^{T}$ is the receiving signal of two users at the sectors of neighbour cells. $B_{I}$ denotes the interfering antenna set. We use zero-forcing beamforming precoding to obtain MIMO multiplexing gain and the precoding matrix is:

$$
\mathbf{M}=\mathbf{H}^{\dagger}
$$

Then the transmitting signal from the remote antenna units becomes:

$$
\mathbf{x}=\mathbf{M s}
$$

where $\mathbf{s} \in \mathbb{C}_{2 \times 1}^{T}$ is the source transmitting data from the antenna units. However, for the sake of a fair comparison with SU-MISO case that the equal transmitting power should be used in comparison, we set the precoding matrix to satify the power constraint as:

$$
\mathbf{M}_{S}=\frac{\mathbf{H}^{\dagger}}{\left\|\mathbf{H}^{\dagger}\right\|}
$$

Thus the receriving signal is given by:

$$
\mathbf{y}=\sqrt{P_{t}} \mathbf{H} \mathbf{M}_{S} \mathbf{s}+\sum_{i \in B_{I}} \sqrt{P_{t}} \mathbf{H}_{i} \mathbf{M}_{S i} \mathbf{s}_{i}+\mathbf{n}
$$

and the MU-MIMO CoMP-Coordinated Beamforming capacity is:

$$
C=B W \log _{2}\left|\mathbf{I}+\frac{P_{t} \mathbf{H}_{S} \mathbf{M}_{S} \mathbf{M}_{S}^{*} \mathbf{H}_{S}^{*}}{\sum_{i \in B_{I}} P_{t} \mathbf{H}_{S} \mathbf{M}_{S} \mathbf{M}_{S}^{*} \mathbf{H}_{S}^{*}+P_{n}}\right|
$$

Assuming that the receiver has perfect channel information, we can use the block diagonalization (BD) algorithm to demodulate the signal by:

$$
\left[\begin{array}{lll}
\mathbf{S} & \mathbf{V} & \mathbf{D}
\end{array}\right]=\operatorname{svd}\left(P_{t} \mathbf{H}_{S} \mathbf{M}_{S} \mathbf{M}_{S}^{*} \mathbf{H}_{S}^{*}\right)
$$

and select the diagonal value of the matrix $\mathbf{V}$ as:

$$
\lambda_{j}=\operatorname{diag}(\mathbf{V})
$$

Thus the downlink capacity of MU-MIMO CB user $k$ is given as:

$$
C_{k}=B W_{k} \log _{2}\left|1+\gamma_{k}^{C B}\right|
$$

where

$$
\gamma_{k}^{C B}=\frac{\prod_{j} \lambda_{j}}{\prod_{i} \lambda_{i}+\delta_{n}^{2}}
$$

The two cooperating antenna units transmit the signal to two users in the neighbour sectors at the same time and same frequency $A_{k}^{O}=A_{k}^{N}$ as illustrated in Fig. 2. To avoid intracell interference the six sectors in a cell are allocated different frequency resources $N_{f}>=6$. We construct the cell topology with a 3-cell cluster consisting of 18 sectors. Considering the sectors and neighbour sectors of cell A, they cover 12 sectors. 6 neighbour sectors are left which can be allocated 3 different frequency resources. Thus to avoid intra-cluster interference $N_{f}>=9$, in this paper we pick $N_{f}=9$. 


\section{B. SU-MISO using Joint Transmission}

Consider SU-MISO transmission, two remote antenna units are communicating with one user. As a form of SU-MISO transmission, the Cooperative Joint Transmission (CJT) uses the Maximum Ratio Transmission (MRT) precoding technique, the receiving signal by a user $k$ is given by [13]:

$$
y_{k}=\sum_{j \in B_{S}} \sqrt{P_{t}} h_{j k} x_{k}+\sum_{i \in B_{I}} \sqrt{P_{t}} h_{i k} x_{j m}+n_{k}
$$

where $n_{k}$ is the AWGN whose power density is $N_{0}$ and $x_{k}$ is the original transmitting signal to user $k$.

The transmitting antenna set is $B_{S}$ and the interfering antenna set is $B_{I}, h_{j k}$ is the channel gain from transmitting antenna $j$ to user $k$.

Thus the downlink Capacity of user $k$ is given as :

$$
C_{k}=B W_{k} \log _{2}\left(1+\gamma_{k}\right)
$$

where

$$
\gamma_{k}=\frac{\sum_{j \in B_{S}} P_{t}\left|h_{j k}\right|^{2}}{\sum_{i \in B_{I}} P_{t}\left|h_{i k}\right|^{2}+P_{n}}
$$

where $P_{n}=N_{0} \times B W$ is the noise power and $B W$ is the bandwidth.

\section{AVERAGE CAPACITY ANALYSIS OF USING LSA}

Due to the geolocation feature of LSA, if we introduce LSA to the MU-MIMO CB C-RAN system for one particular cell, this cell will obtain more spectrum. However, the cell edge users have to quit from MU-MIMO CB and use SUMISO JT in LSA band, since the neighbour cell users are not allowed to use LSA spectrum. Moreover, the previous paired and cooperated users in the neighbour cell will also have to quit from MU-MIMO CB and use SU-MISO JT in MNO band. We compare the performance between using LSA and not using LSA by comparing the average downlink capacity of these two scenarios and use the comparison results for decision making on usage of LSA. The comparison problem is divided into three parts:

1) Downlink capacity analysis of MNO users using MUMIMO CB, LSA cell edge users using SU-MISO JT and previous paired MNO users using SU-MISO JT;

2) Average capacity comparison and threshold for using LSA or not;

3) Average capacity gain between using LSA or not.

\section{A. Downlink Capacity Analysis for Cell Edge Users}

Assume $N_{L S A}$ numbers of users in the LSA cell such as the central cell A in Fig. 2 are switched from previous band to LSA band. Assume all remote antenna units support both MNO band and LSA band. The number of paring users is equal to the number of LSA user according to our user paring assumption.

The average capacity of CB transmission is given as:

$$
E\left[C^{C B}\right]=\frac{\sum_{k=1}^{N_{U E}} C_{k}^{C B}}{N_{U E}}=\frac{B W_{e d g e}}{9} \log _{2}\left(1+E\left[\gamma^{C B}\right]\right)
$$

The average capacity of LSA transmission is given as:

$$
\begin{aligned}
& E\left[C^{L S A}\right]=\frac{1}{N_{U E}} \sum_{k=1}^{N_{U E}} C_{k} \\
& =\frac{\sum_{k=1}^{N_{L S A}} C_{k}^{J T_{0}}+\sum_{k=1}^{N_{L S A}} C_{k}^{J T_{1}}+\sum_{k=1}^{N_{U E}-2 N_{L S A}} C_{k}^{C B}}{N_{U E}} \\
& =\frac{N_{L S A}\left(E\left[C^{J T_{0}}\right]+E\left[C^{J T_{1}}\right]\right)+\left(N_{U E}-2 N_{L S A}\right) E\left[C^{C B}\right]}{N_{U E}} \\
& =\alpha\left(E\left[C^{J T_{0}}\right]+E\left[C^{J T_{1}}\right]\right)+(1-2 \alpha) E\left[C^{C B}\right]
\end{aligned}
$$

where $C^{J T_{0}}$ is the average SU-MISO JT capacity of users in the LSA cell edge and $C^{J T_{1}}$ is the average SU-MISO JT capacity of previous paired users in the sectors of neighbour cells. We use $\alpha=\frac{N_{L S A}}{N_{U E}}$ to denote the ratio between the number of LSA users over the number of all users.

We consider a snapshot of the random located users, for one user $k$ in the LSA cell edge, the downlink SU-MIMO JT capacity is given as:

$$
C_{k}^{J T_{0}}=B W_{k}^{L S A} \log _{2}\left(1+\gamma_{k}^{J T_{0}}\right)
$$

where $B W_{k}^{L S A}=\frac{B W^{L S A}}{6}$. We assume only cell $\mathrm{A}$ is in the LSA zone and there are no other cells using the same frequency resource in the LSA band with cell $\mathrm{A}$, thus there is no interference from other cells for LSA users in our model.

$$
\gamma_{k}^{J T_{0}}=\frac{\sum_{j \in B_{S}} P_{t}\left|h_{j k}\right|^{2}}{P_{n}}
$$

However for one user $k$ the previous paring users in the sectors of neighbour cells, there are interfering signals from other cells using the same frequency slices, thus the downlink SU-MISO capacity considering ICI is given as:

$$
C_{k}^{J T_{1}}=B W_{k}^{J T_{1}} \log _{2}\left(1+\gamma_{k}^{J T_{1}}\right)
$$

where $B W_{k}^{J T_{1}}=B W_{k}^{C B}=\frac{B W_{\text {edge }}}{N_{f}}$, in this paper $N_{f}=9$ and

$$
\gamma_{k}^{J T_{1}}=\frac{\sum_{j \in B_{S}} P_{t}\left|h_{j k}\right|^{2}}{\sum_{i \in B_{I}} P_{t}\left|h_{i k}\right|^{2}+P_{n}}
$$

Thus the average capacity of all cell edge users with some users using LSA bandwidth is given as:

$$
\begin{aligned}
& E\left[C^{L S A}\right]= \\
& \alpha\left(\frac{B W_{L S A}}{6} \log _{2}\left(1+E\left[\gamma^{J T_{0}}\right]\right)+\frac{B W_{\text {edge }}}{9} \log _{2}\left(1+E\left[\gamma^{J T_{1}}\right]\right)\right) \\
& \quad+(1-2 \alpha) \frac{B W_{\text {edge }}}{9} \log _{2}\left(1+E\left[\gamma^{C B}\right]\right) \\
& =B W_{\text {edge }}\left(\alpha\left(\frac{\eta}{6} \log _{2}\left(1+E\left[\gamma^{J T_{0}}\right]\right)+\frac{1}{9} \log _{2}\left(1+E\left[\gamma^{J T_{1}}\right]\right)\right)\right. \\
& \left.\quad+(1-2 \alpha) \frac{1}{9} \log _{2}\left(1+E\left[\gamma^{C B}\right]\right)\right)
\end{aligned}
$$

where $\eta=\frac{B W_{L S A}}{B W_{\text {edge }}}$ denotes the ratio between the LSA bandwidth and the MNO cell edge bandwidth. Moreover, the user ratio $\alpha$ and the bandwidth ratio $\eta$ is independent with $E\left[\gamma^{J T_{0}}\right], E\left[\gamma^{J T_{1}}\right]$ or $E\left[\gamma^{C B}\right]$. 


\section{B. Average Dowlink Capacity Comparison}

Our target is using LSA to obtain certain increase in the performance, thus the problem is find out when we can obtain higher average capacity with LSA bandwidth than original MNO bandwidth, then we can make the decision whether to use LSA bandwidth and how much bandwidth we need to fulfill the capacity requirements. We compare (21) and (15) and it leads to:

$$
\begin{aligned}
& \alpha\left(3 \eta \log _{2}\left(1+E\left[\gamma^{J T_{0}}\right]\right)+2 \log _{2}\left(1+E\left[\gamma^{J T_{1}}\right]\right)\right. \\
& \left.\geq 4 \alpha \log _{2}\left(1+E\left[\gamma^{C B}\right]\right)\right) \\
& 3 \eta \log _{2}\left(1+E\left[\gamma^{J T_{0}}\right]\right)+2 \log _{2}\left(1+E\left[\gamma^{J T_{1}}\right]\right. \\
& \left.\geq 4 \log _{2}\left(1+E\left[\gamma^{C B}\right]\right)\right)
\end{aligned}
$$

Assume $E\left[\gamma^{J T_{0}}\right], E\left[\gamma^{J T_{1}}\right]$ and $E\left[\gamma^{C B}\right]$ are all far greater than one, then we use $\log _{2}(1+a) \approx \log _{2}(a), a \gg 1$ to obtain an approximation of (22) given as :

$$
\eta \geq \frac{4 \log _{2}\left(E\left[\gamma^{C B}\right]\right)-2 \log _{2}\left(E\left[\gamma^{J T_{1}}\right]\right)}{3 \log _{2}\left(E\left[\gamma^{J T_{0}}\right]\right)}
$$

Thus the threshold of $\eta$ is given as:

$$
\eta *=\frac{4 E\left[\gamma_{d B}^{C B}\right]-2 E\left[\gamma_{d B}^{J T_{1}}\right]}{3 E\left[\gamma_{d B}^{J T_{0}}\right]}
$$

\section{Average Downlink Capacity Gain}

Assume we have enough LSA bandwidth with $\eta>\eta *$, the performance gain on LSA over the original MU-MIMO MNO is given as :

$$
\begin{aligned}
& G=\frac{E\left[C^{L S A}\right]}{E\left[C^{C B}\right]}=\left(B W _ { \text { edge } } \left(\alpha \left(\frac{\eta}{6} \log _{2}\left(1+E\left[\gamma^{J T_{0}}\right]\right)\right.\right.\right. \\
& \left.\left.\left.+\frac{1}{9} \log _{2}\left(1+E\left[\gamma^{J T_{1}}\right]\right)\right)+\frac{(1-2 \alpha)}{9} \log _{2}\left(1+E\left[\gamma^{C B}\right]\right)\right)\right) \\
& /\left(\frac{B W_{\text {edge }}}{9} \log _{2}\left(1+E\left[\gamma^{C B}\right]\right)\right) \\
& =\left(\alpha\left(3 \eta \log _{2}\left(1+E\left[\gamma^{J T_{0}}\right]\right)+2 \log _{2}\left(1+E\left[\gamma^{J T_{1}}\right]\right)\right)\right. \\
& \left.+2(1-2 \alpha) \log _{2}\left(1+E\left[\gamma^{C B}\right]\right)\right) /\left(2 \log _{2}\left(1+E\left[\gamma^{C B}\right]\right)\right) \\
& \approx \alpha \eta\left(\frac{3 E\left[\gamma_{d B}^{J T_{0}}\right]}{2 E\left[\gamma_{d B}^{C B}\right]}\right)+(1-2 \alpha)+\frac{E\left[\gamma_{d B}^{J T_{1}}\right]}{E\left[\gamma_{d B}^{C B}\right]}
\end{aligned}
$$

We use $A$ to denote the ratio between SNR of SU-MISO in LSA users over the SNR of the MU-MIMO users $A=\frac{E\left[\gamma_{d B}^{\gamma}\right]}{E\left[\gamma_{d B}^{C B}\right]}$; and $B$ to denote the ratio between SNR of SU-MISO in MNO users over the SNR of the MU-MIMO users $B=\frac{E\left[\gamma_{d B}^{T T_{1}}\right]}{E\left[\gamma_{d B}^{C B}\right]}$. Due to $E\left[\gamma_{d B}^{J T_{0}}\right]>E\left[\gamma_{d B}^{C B}\right]>E\left[\gamma_{d B}^{J T_{1}}\right]$, we have $A>1$ and $B<1$, then (25) is given as

$$
G \approx\left(\frac{3 \eta A}{2}-2\right) \alpha+B+1
$$

Moreover

$$
\frac{3 \eta A}{2} \geq \frac{4 E\left[\gamma_{d B}^{C B}\right]-2 E\left[\gamma_{d B}^{J T_{1}}\right]}{3 E\left[\gamma_{d B}^{J T_{0}}\right]} \frac{3 E\left[\gamma_{d B}^{J T_{0}}\right]}{2 E\left[\gamma_{d B}^{C B}\right]}>2
$$

Thus for a fixed LSA bandwidth ratio $\eta>\eta *$ in our system model, the performance gain will have a linear increment with the increase of the LSA users' ratio. where $0<\alpha<=\frac{N_{U E_{c e l l}}}{N_{U E}}$.

\section{NumericAl Results}

The channel model in the simulations includes the small scale fading channel and the large scale fading channel which is the path loss. Shadow fading is not considered because it is assumed that for a cellular network shadow fading from the antenna to UE is a constant value. The channel gain is given by $h_{j}=g_{j} * \sqrt{\Omega_{j}}$ [14], where $g_{j}$ is the small-scale channel gain which is modeled as a Rayleigh channel and $\Omega_{j}$ is the multiplication of the path loss and shadow fading from antenna $j$ to the UE in which only path loss $L_{j}$ has been taken into consideration in this paper.

The scenario considered in this paper is the cellular network C-RAN, thus the path loss parameters that configured for the simulation are transmitting power $P_{t}=43 \mathrm{dBm}, d_{0}=100$ $\mathrm{m}$ and $\beta=4[15]$.

\section{A. Capacity Cumulative Distribution Function}

In our previous work [16], we proved the noise power is a key factor for downlink capacity in FFR-DAS. Therefore, in this paper, we consider none-zero AWGN noise power $P_{n}=-72.8 \mathrm{dBm}$. The downlink average capacity of the cell boundary users with the noise power $P_{n}$ is given as: the capacity Cumulative Distribution Function (CDF) is investigated in this paper as follows. The average capacity for a user at the cell boundary is given by:

$$
\begin{gathered}
C=\frac{1}{2 \pi} \int_{0}^{2 \pi} \int_{0}^{\infty} \log _{2}(1+\gamma) f_{\Gamma_{N}\left(r_{e}, \theta_{e}\right)}(\gamma) d \gamma d \theta_{e} \\
=\frac{1}{\pi / 6} \int_{0}^{\pi / 6} \int_{0}^{\infty} \log _{2}(1+\gamma) f_{\Gamma_{N}\left(r_{e}, \theta_{0}\right)}(\gamma) d \gamma d \theta_{0}
\end{gathered}
$$

where $\left.r_{e}=\frac{\sqrt{3}}{2} R_{0} \cos ^{-1}\left(\theta_{0}-\frac{\pi}{6}\right)\right)$ and $\gamma$ is equal to $\gamma^{J T 0}$, $\gamma^{J T 1}$ and $\gamma^{C B^{2}}$ in (18), (20) and (11) respectively.

We use Monte-Carlo simulation with $\eta=\eta *$ and the results are shown in Fig. 3. The results show LSA SU-MISO JT users' average capacity is higher than the MNO MIMO-CB users and higher than MNO SU-MISO JT users. This validate our analysis that using LSA in MU-MIMO CB systems will cause a capacity decrease for the previous paired users in the sectors of the neighbouring cells.

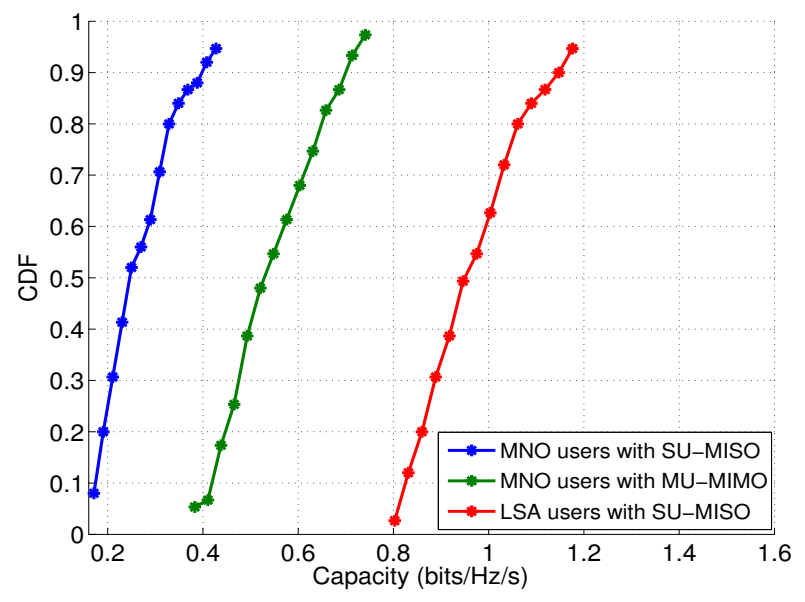

Fig. 3: Capacity CDF. 


\section{B. Bandwidth Ratio Threshold}

We simulate the average capacity using LSA bandwidth and only MNO bandwidth with different values of $\eta$ and two values of $\alpha$ using our system and channel model. The results are shown in Fig. 4 and that shows the threshold on $C^{L S A} \geq$ $C^{C B}$ is dependent on $\eta$ but independent with $\alpha$. Moreover, the threshold is close to the analytical result we derived in (24).

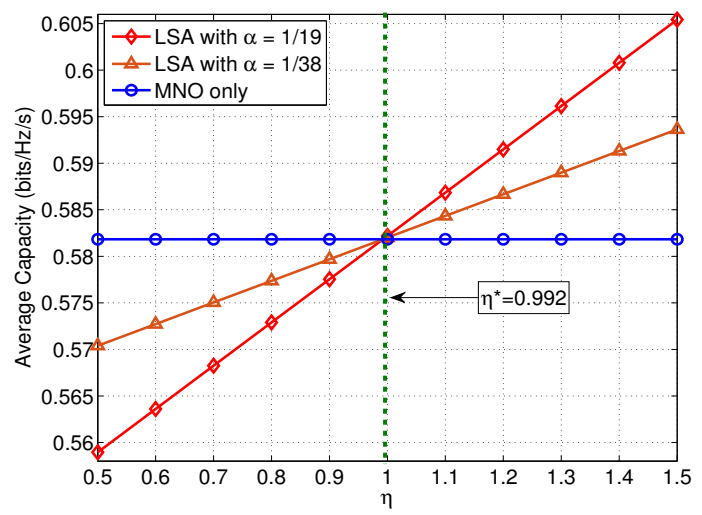

Fig. 4: Average Capacity Comparison.

\section{Average Downlink Capacity Gain}

We derived the capacity gain between LSA and MNO with the numerical results shown in Fig. 5. With the increase of $\eta$, the capacity gain will also increase. This shows that MNO use more LSA bandwidth, and hence the average LSA capacity is increased in frequency domain. For a fixed value of $\eta$, the capacity gain has a linear increment with the increase of $\alpha$. This shows that more users join the LSA bandwidth for a longer time, and hence the LSA capacity is increased in the time domain.

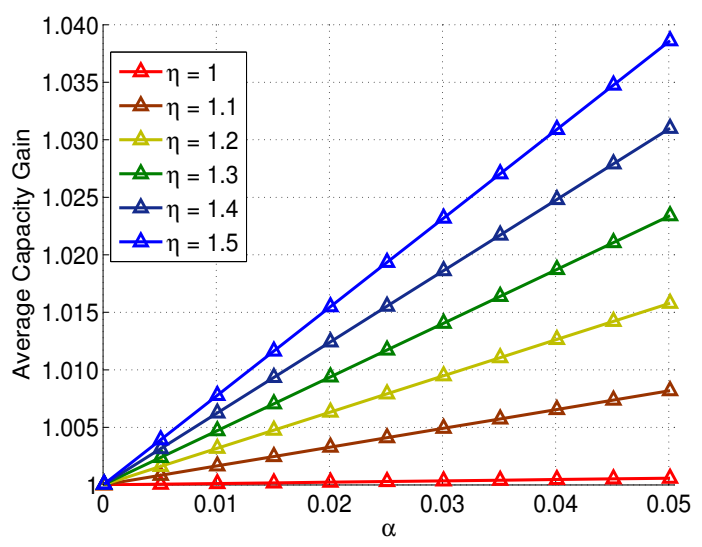

Fig. 5: Average Capacity Gain with LSA users' ratio.

\section{CONCLUSION}

In this paper, we use the Licensed Shared Access concept in the analysis of the downlink capacity of cell edge users in Distributed Antenna Systems for 5G wireless communication with the Network Virtualization concept using Fractional Frequency Reuse scheme for resource allocation. We analyzed and compared the capacity for using LSA with SU-MISO joint transmission with original MNO MU-MIMO coordinated beamforming. A threshold of the LSA bandwidth ratio for the average capacity and the average capacity gain are derived. Numerical results validate the analytical results. The analytical results provide a merit for the desicion making of using LSA in $5 \mathrm{G}$ wireless communication systems in the context of Network Virtualization such as C-RAN without the need for simulation.

\section{ACKNOWLEDGEMENT}

Ying He is a recipient of a Macquarie University Research Excellence Scholarship.

\section{REFERENCES}

[1] A. M. Saleh, A. J. Rustako, and R. S. Roman, "Distributed antenna for indoor radio communications," IEEE Trans. Commun., vol. 35, no. 3, pp. 1245-1251, Feb. 1987.

[2] L. Dai, S. Zhou, and Y. Yao, "Capacity analysis in cdma distributed antenna systems," IEEE Trans. Wireless Commun., vol. 4, no. 6, pp. 2613-2620, Nov. 2005.

[3] S. Bhaumik, S. Chandrabose, M. Jataprolu, G.Kumar, A. Muralidhar, P. Polakos, V. Srinivasan, and T. Woo, "Cloudiq: A framework for processing base stations in a data center," in Mobicom, 2012, pp. 125136.

[4] China Mobile Research Institute, "C-ran the road towards green ranwhite paper," Oct. 2011, http://labs.chinamobile.com/cran/wp-content/ uploads/CRAN_white_paper_v2_5_EN(1).pdf.

[5] X. Costa-Perez, J. Swetina, T. Guo, R. Mahindra, and S. Rangarajan, "Radio access network virtualization for future mobile carrier networks," IEEE Comm. Magzine, vol. 51, no. 7, 2013.

[6] Y. He, E. Dutkiewicz, G. Fang, and J. Shi, "Downlink capacity in cloud radio access networks with fractional frequency reuse," in WPMC2014.

[7] Y. Zhou, J. Wang, and M. Sawahashi, "Downlink transmission of broadband ofcdm systems-part i: Hybrid detection," Communications, IEEE Transactions on, vol. 53, no. 4, pp. 718-729, 2005.

[8] J. Thompson, X. Ge, H.-C. Wu, R. Irmer, H. Jiang, G. Fettweis, and S. Alamouti, "5g wireless communication systems: Prospects and challenges," Communications Magazine, IEEE, vol. 52, no. 2, pp. 6264, 2014.

[9] E. R. 205, "Licensed shared access," 2014.

[10] M. Palola, M. Matinmikko, J. Prokkola, M. Mustonen, M. Heikkila, T. Kippola, S. Yrjola, V. Hartikainen, L. Tudose, A. Kivinen et al., "Live field trial of licensed shared access (1sa) concept using lte network in 2.3 ghz band," in Dynamic Spectrum Access Networks (DYSPAN), 2014 IEEE International Symposium on. IEEE, 2014, pp. 38-47.

[11] M. Matinmikko, H. Okkonen, M. Palola, S. Yrjola, P. Ahokangas, and M. Mustonen, "Spectrum sharing using licensed shared access: the concept and its workflow for lte-advanced networks," Wireless Communications, IEEE, vol. 21, no. 2, pp. 72-79, 2014.

[12] M. Mustonen, T. Chen, H. Saarnisaari, M. Matinmikko, S. Yrjola, and M. Palola, "Cellular architecture enhancement for supporting the european licensed shared access concept," Wireless Communications, IEEE, vol. 21, no. 3, pp. 37-43, 2014.

[13] Lo and T. KY, "Maximum ratio transmission," in Communications, 1999. ICC'99. 1999 IEEE International Conference on, vol. 2. IEEE, 1999, pp. 1310-1314.

[14] W. Choi and J. G. Andrews, "Downlink performance and capacity of distributed antenna systems in a multicell environment," Wireless Communications, IEEE Transactions on, vol. 6, no. 1, pp. 69-73, 2007.

[15] A. Goldsmith, Wireless communications. Cambridge university press, 2005.

[16] Y. He, E. Dutkiewicz, G. Fang, and J. Shi, "Differential capacity bounds for distributed antenna systems under low snr conditions," in IEEE ICC2014. 